\title{
ЦИТ: ua117-052
}

DOI: $10.21893 / 2415-7538.2016-05-1-052$

УДК 512.543

МІНІМАЛЬНІ СИСТЕМИ ТВІРНИХ ДЛЯ

Сікора B.C.

\section{ГІПЕРОКТАЕДРАЛЬНОЇ ГРУПИ ТА ЇЇ НОРМАЛЬНИХ ПІДГРУП}

Чернівецький національний університет імені Юрія Федьковича,

Україна, м. Чернівці, вул. Коцююбинського, 2, 58000

\section{MINIMAL GENERATORS SYSTEMS FOR \\ THE HYPEROKTAHEDRAL GROUP AND ITS NORMAL SUBGROUPS}

Sikora V.S.

Chernivtsi National University named Yurij Fedkovych,

Chernivtsi, vul. Kotsybynskogo, 2, 58000

Анотація. Побудовано приклади мінімальних (щодо кількості елементів) систем твірних для нормальних дільників гіпероктаедральної групи.

Ключові слова: гіпероктаедральна група, вінцевий добуток, система твірних.

Abstract. The minimal (on quantity of elements) systems of generators are constructed for the normal subgroups of hyperoctahedral group.

Key Words: hyperoctahedral group, wreath product, system of generators.

Вступ та огляд літератури. Гіпероктаедральна група (група симетрій $n_{-}$ вимірного куба) - це група тих лінійних перетворень евклідового простору $E_{n}$, які діють на векторах ортонормованого базису $\bar{e}_{1}, \bar{e}_{2}, \ldots, \bar{e}_{n}$ цього простору перестановками або замінами напрямків (тобто заміною $\bar{e}_{i}$ на $-\bar{e}_{i}$ ). Іншими словами, гіпероктаедральну групу можна розглядати як "найбільшу" кристалографічну групу гіперкубічної гратки, яку визначають як групу, породжену всіма лінійними комбінаціями вигляду $\bar{x}=\sum_{k=1}^{n} x^{k} \bar{e}_{k}$, де $x^{k}-$ інтегральні коефіцієнти та $\bar{e}_{1}, \bar{e}_{2}, \ldots, \bar{e}_{n}$ - стандартний ортонормований базис $n$-вимірного евклідового простору $E_{n}$. Ця група має порядок $n ! 2^{n}$ i, як абстрактна група, ізоморфна вінцевому добутку $S_{n} Z_{2}$ симетричної групи $S_{n}$ степеня $n$ на циклічну групу $Z_{2}$. Гіпероктаедральні групи є одним з прикладів груп Вейля (типу $B_{n}$ ) [1]. Основні відомості про будову гіпероктаедральних груп зібрано в оглядовій статті [2]. У роботі [3] побудовано мінімальні (щодо кількості елементів) системи твірних групи $H_{n}=S_{n} Z_{2}$ (яку також називатимемо гіпероктаедральною). Дану роботу присвячено побудові аналогічних систем твірних для нормальних дільників групи $H_{n}$.

Постановка задачі та методи. Елементами групи $H_{n} \in$ підстановки, які можна зображати у вигляді таблиць $u=\left[\sigma ; \alpha_{1}, \alpha_{2}, \ldots, \alpha_{n}\right]$, де $\sigma \in S_{n}$ 一 деяка під- 
становка множини $\{1,2, \ldots, n\} ; \alpha_{i} \in Z_{2}=\{\overline{0}, \overline{1}\}$ (див. [3]). Добуток двох таблиць $u$ та $v=\left[\tau ; \beta_{1}, \beta_{2}, \ldots, \beta_{n}\right]$ визначається рівністю

$$
u \cdot v=\left[\sigma \cdot \tau ; \alpha_{1}+\beta_{\sigma(1)}, \alpha_{2}+\beta_{\sigma(2)}, \ldots, \alpha_{n}+\beta_{\sigma(n)}\right],
$$

де “ + ” означає додавання в $Z_{2}$, а оберненою до $u$ буде таблиця

$$
u^{-1}=\left[\sigma^{-1} ; \alpha_{\sigma^{-1}(1)}, \alpha_{\sigma^{-1}(2)}, \ldots, \alpha_{\sigma^{-1}(n)}\right] \text {. }
$$

Опишемо нормальні дільники групи $H_{n}=S_{n} Z_{2}$.

1) Тривіальними нормальними дільниками в гіпероктаедральній групі $H_{n}$ будуть вона сама і підгрупа

$$
L=\{[\varepsilon ; \alpha, \alpha, \ldots, \alpha] \mid \alpha=\overline{1} \text { або } \alpha=\overline{0}\}=<[\varepsilon ; \overline{1}, \overline{1}, \ldots, \overline{1}]>
$$

тобто $H_{n \unlhd} H_{n \text { та } L \unlhd} H_{n}$.

2) $G_{n}=A_{n \chi} Z_{2}=\left\{\left[\lambda ; \alpha_{1}, \alpha_{2}, \ldots, \alpha_{n}\right] \mid \lambda \in A_{n}, \alpha_{i} \in Z_{2}, i=\overline{1, n}\right\}_{\unlhd} S_{n \chi} Z_{2}$.

3) $W=\left\{\left[\pi ; \alpha_{1}, \alpha_{2}, \ldots, \alpha_{n}\right] \mid \pi \in S_{n}, \alpha_{i} \in Z_{2}, i=\overline{1, n}, \sum_{i=1}^{n} \alpha_{i}=\overline{0}\right\}_{\unlhd} S_{n} Z^{2}$.

Неважко переконатися, що група $W$ ізоморфна групі Вейля типу $D_{n}$.

$$
M=\left\{\left[\lambda ; \alpha_{1}, \alpha_{2}, \ldots, \alpha_{n}\right] \mid \lambda \in A_{n}, \alpha_{i} \in Z_{2}, i=\overline{1, n}, \sum_{i=1}^{n} \alpha_{i}=\overline{0}\right\}_{\unlhd} A_{n} Z_{2},
$$

причому $M \unlhd W$.

5)

$K=\left\{\left[\varepsilon ; \alpha_{1}, \alpha_{2}, \ldots, \alpha_{n}\right] \mid \varepsilon \in A_{n}, \alpha_{i} \in Z_{2}, i=\overline{1, n}\right\}_{\unlhd} A_{n} Z_{2}$

6)

$$
T=\left\{\left[\varepsilon ; \alpha_{1}, \alpha_{2}, \ldots, \alpha_{n}\right] \mid \varepsilon \in A_{n}, \alpha_{i} \in Z_{2}, i=\overline{1, n}, \sum_{i=1}^{n} \alpha_{i}=\overline{0}\right\}_{\unlhd} K_{\text {та }} T \unlhd M .
$$

Зауважимо, що описана вище підгрупа $L$ є нормальним дільником в $T$.

Наведемо деякі допоміжні твердження.

Лема 1 [4]. Підстановки $a=(1,2, \ldots, k)_{m a} b=(1,2)$ утворюють базу симетричної групи $S_{k}(k \geq 3)$.

Лема 2 [5]. Пара підстановок $a=(1,2, \ldots, k)_{m a} c=(1,2, \ldots, m)$, де $2 \leq m<k$, буде базою симетричної групи $S_{k}$ (якщуо хоча б одна з підстановок а чи $\epsilon_{\epsilon}$ непарною), або знакозмінної групи $A_{k}$ (якщуо обидві підстановки $a, c \in$ парними).

Лема 3 [6]. Для довільного непарного $k \geq 3$ знакозмінна група $A_{k}$ породжусться підстановками $(1,2, \ldots, k),(1,2,3)$ або $(1,2, \ldots, k),(3,4, \ldots, k)$ (остання пара при $k \geq 5)$, а при довільному парному $k \geq 4$ - підстановками $(1,2, \ldots, k-1)$, $(2,3, \ldots, k)$. 
Мінімальні системи твірних групи $H_{n}=S_{n} Z_{2}$ побудовано в [3]. Метою статті $є$ побудова мінімальних систем твірних для нормальних дільників гіпероктаедральної групи $H_{n}$. Група $L$ містить лише дві підстановки $[\varepsilon ; \overline{1}, \overline{1}, \ldots, \overline{1}]$ та $[\varepsilon ; \overline{0}, \overline{0}, \ldots, \overline{0}]$ - i $\epsilon$ циклічною, породженою елементом $[\varepsilon ; \overline{1}, \overline{1}, \ldots, \overline{1}]$. Тобто мінімальна система твірних групи $L$ складається 3 однієї підстановки $h=[\varepsilon ; \overline{1}, \overline{1}, \ldots, \overline{1}]$.

Доведено наступні 5 тверджень.

Теорема 1. Для довільного натурального $n \geq 4$ мінімальними (щодо кількості елементів) системами твірних групи $A_{n} Z{ }_{2}$ є наступні:

a) $\left\{u_{1}, u_{2}\right\}$, якщзо $n \geq 5$ - непарне, де

$$
u_{1}=[(1,2, \ldots, n) ; \overline{1}, \overline{1}, \ldots, \overline{1}], \quad u_{2}=[(3,4, \ldots, n) ; \overline{0}, \overline{0}, \overline{1}, \overline{0}, \ldots, \overline{0}] ;
$$

б) $\left\{v_{2}, v_{2}\right\}$, якщо $n \geq 4$ - парне, де

$$
v_{1}=[(1,2, \ldots, n-1) ; \overline{1}, \overline{0}, \ldots, \overline{0}], \quad v_{2}=[(2,3, \ldots, n) ; \overline{0}, \overline{0}, \ldots, \overline{0}] .
$$

Зауваження. При побудові мінімальних систем твірних групи $G_{n}=A_{n} Z_{2}$ випадки $n=2$ і $n=3$ нами не розглядаються, як тривіальні, оскільки знакозмінні групи $A_{2}$ та $A_{3}$ - циклічні.

Розглянемо тепер групу $W \unlhd S_{n} Z_{2}$. Має місце твердження.

Теорема 2. Для довільного $n \geq 4$ група $W$ e 2-породженою.

Розглянемо групу $M$, визначену вище, яка $\epsilon$ нормальним дільником в групах $A_{n} Z_{2}$ та $W$.

Теорема 3. Для довільного $n \geq 5$ група $M \in$ 2-породженою.

Теорема 4. Група $K=\left\{\left[\varepsilon ; \alpha_{1}, \alpha_{2}, \ldots, \alpha_{n}\right] \mid \varepsilon \in A_{n}, \alpha_{i} \in Z_{2}, i=\overline{1, n}\right\}$ для довіль-

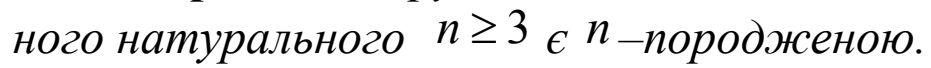

Теорема 5. Група $T$ для довільного натурального $n \geq 3 \epsilon^{(n-1)}$ породженою.

Висновки. Побудовано приклади мінімальних (щодо кількості елементів) систем твірних для нормальних дільників гіпероктаедральної групи. Отримані результати можна використовувати при побудові незвідних систем твірних вінцевих добутків скінченної (чи нескінченної) кількості симетричних, знакозмінних і циклічних груп та у нормальних підгрупах таких груп.

Література:

1. Бурбаки Н. Группы и алгебры Ли. - М.: Мир, 1968. — 331с.

2. Baake M. Structure and representations of the hyperoctahedral group // J. Math. Phys. - 25 (11), November 1984. - P.3171-3182.

3. Сікора В.С. Двоелементні бази гіпероктаедральних груп // Вісник Київського університету. Серія фіз.-мат. науки.- 1999.- Вип.1.- С.87-93. 
4. Калужнин Л.А., Сущанский В.И. Преобразования и перестановки. - М.: Наука, 1985. - 112c.

5. Isaacs I.M., Thilo Zieschang. Generating Symmetric Groups // Math. Notes. - 10 (1995).- P.734-738.

6. Пикар С. О базисах симметрической группы // Кибернетический сборник, Москва: Мир, 1965.- Вып. 1.- с.7-34.

7. Заводя М.В., Сікора В.С. Системи твірних нормальних підгруп гіпероктаедральної групи.- Вісн. Київськ. ун-ту. Серія: Фізико-математичні науки.2002.- N3.- C. 28-34.

8. Сікора В.С. Побудова мінімальних систем твірних для гіпероктаедральної групи та iї нормальних підгруп // Сборник научных трудов SWorld.-2015.- Том 21.- Вып. 1(38).- C.85-91.

Стаття відправлена: 31.03.2017 p.

(C) Сікора В.C. 